\title{
Editorial
}

\author{
Axel Ruhe
}

Published online: 8 November 2012

(C) Springer Science+Business Media Dordrecht 2012

\section{Introduction to the contents of issue 52:4}

The issue you now have in your hand, or on your screen, shows that BIT is becoming successively more present in the Scientific Computation area. We have three papers that deal with computational solution of magnetohydrodynamical, MHD, problems, and two more contributions of a computational physics flavor.

In the first paper, Lyonell Boulton and Michael Strauss derive certified enclosures for eigenvalues of linear MHD operators, in the practically interesting plane slab and cylindrical pinch configurations. Schur complements are needed for the computation. The position of the essential spectrum makes this more complicated than finding eigenvalue bounds for matrix problems.

A related problem is treated in the paper by Paolo Corti and Siddhartha Mishra who study algorithms for the magnetic induction equations with Hall Effect. These equations are non-linear and include third-order spatial and spatio-temporal mixed derivatives. They develop stable finite difference schemes that preserve the energy bounds for the continuous problem.

In the third MHD related paper, Holger Heumann, Ralf Hiptmair, Kun Li, and Jinchao $\mathrm{Xu}$ study fully discrete semi-Lagrangian methods for advection dominated differential forms. These occur as building blocks for MHD models.

In another physics paper, Haotao Cai develops a fast solution algorithm for the Dirichlet problem of the Helmholtz equation in two dimensions, by solving a boundary integral equation of the first kind. It gives a sparse matrix and the cost of its solution grows with a linear bound when a Fourier Galerkin algorithm is used.

In the final physics paper, David Cohen and Ludwig Gauckler solve the cubic time dependent Schrödinger equation with spectral discretization in space and an

A. Ruhe $(\bowtie)$

School of Science (SCI), Royal Institute of Technology (KTH), 10044 Stockholm, Sweden

e-mail: ruhe@kth.se 
exponential integrator in time. They show how to keep energy, mass and momentum constant along the numerical solution over long times.

We have three contributions in this issue to Numerical Linear Algebra:

Zvonimir Bujanović and Zlatko Drmač study a block two sided Jacobi, or more properly Kogbetliantz, method for computing the singular value decomposition, SVD, of a matrix. Global convergence and asymptotic quadratic convergence is established, which makes a parallel implementation feasible.

Cedric Effenberger and Daniel Kressner develop an algorithm for an eigenvalue problem that is nonlinear in the eigenvalue parameter. They approximate the nonlinear function with a Chebyshev interpolation polynomial, whose eigenvalue problem can be solved by the usual Krylov subspace methods.

Miroslav Rozložník, Miroslav Ti̊ma, Alicja Smoktunowicz, and Jiři Kopal study the numerical stability of orthogonalization methods where the inner product is induced by a nontrivial symmetric positive definite matrix. Errors caused by finite precision arithmetic in the orthogonalization and reorthogonalization are analyzed.

We have two contributions in Numerical Approximation related to computer aided geometric design, CAGD:

Xuli Han and Yuanpeng Zhu describe Bezier curves based on trigonometric functions. Such curves may be better than the standard polynomials, when adjusting shape and fitting to control points.

Marie-Laurence Mazure gives a new criterion to decide whether a spline space is useful for CAGD. She studies splines with pieces taken from different Extended Chebyshev spaces, that satisfy parametric continuity conditions at the knots.

Finally we have one paper that deals with the inner work of a computational algorithm:

Siegfried M. Rump studies a consistent set of rules for interval arithmetic with finitely many endpoints. The end points are taken from the finite set of representable numbers, in e.g. an IEEE 754 system, but intervals may have either open or closed ends, which allows handling of converging sequences with a limit.

\section{Editorial board}

We thank Per Christian Hansen who leaves the board after ten years service. He has given BIT presence in his image processing specialty and on the Danish scene, concluded with the nice BIT Circus last summer in Copenhagen.

We welcome three new board members, Rosemary Renaut from Arizona State University, Raul Tempone, King Abdullah University of Science and Technology, Saudi Arabia, previously at KTH in Stockholm, and Michael Vogelius, Rutgers University, New Jersey, USA. We already know you as members of the BIT family, now we look forward to your help to make BIT present in your specialties and in your circles! 


\section{Referees}

Let me end this, my tenth year as your Editor in Chief, by saying thank you to all referees that have helped BIT and the authors to keep our quality. You are anonymous to the authors but we acknowledge your fundamental help to BIT.

These are present in the BIT files this year. Forgive me, if I have lost somebody deserving to be on this list:

Lidia Aceto

Rachid Ait-Haddou

Ishtiaq Ali

Martin Alnæs

Bradley Alpert

Gil Ariel

Martin Arnold

Vladislav Babenco

Zheng-Jian Bai

Zhong-Zhi Bai

Domingo Barrera

Dan Bates

Henning Behnke

Alex Bespalov

Sergio Blanes

Shreemayee Bora

Oscar Borries

Hermann Brunner

Erik Burman

Christine Böckmann

Martin Campos Pinto

Xiao-Wen Chang

Paolo Costantini

Hua Dai

Oleg Davydov

Thomas K. DeLillo

Huaian Diao

Victor Dominguez

Froilan Dopico

Philipp Dorsek

Zlatko Drmač

Jérôme Droniou

Ricardo Duran

Lars Eldén

Wayne Enright
Walter Gautschi

Mike Giles

Stef Graillat

Martin Gutknecht

Johnny Guzman

Thomas Hagstrom

Eskil Hansen

Vjeran Hari

Ken Hayami

Adrian T. Hill

Johan Hoffman

Zhongyi Huang

Martin Hutzenthaler

Daan Huybrechs

Elias Jarlebring

Ming Jiang

Pavel Jiranek

Patrick Joly

Mika Juntunen

Michael Karow

David I. Ketcheson

David Kincaid

Martin Kleinsteuber

Othmar Koch

Natasa Krejiç

Bishnu Lamichhane

Mats Larson

Stig Larsson

Hui-Yuan Li

Ren-Cang Li

Cecilia Magherini

Stephen Mann

Carla Manni

José Mario Martínez

Günter Mayer 
Robert McLachlan

William McLean

Karl Meerbergen

Jean-Louis Merrien

Gerard Meurant

Carl Christian Kjelgaard Mikkelsen

Juan Monterde

Axel Målqvist

David Nicholls

Datian Niu

Jan Nordström

Sotirios E. Notaris

Mario Ohlberger

Gabriel Oksa

Luke Olson

Christoph Ortner

J.M. Pena

Roger Pettersson

Helmut Podhaisky

John D. Pryce

Lyle Ramshaw

Christoph Reisinger

Sara Remogna

Arnold Reusken

Robert N. Rieben

Hans Riesel

Giuseppe Rodriguez

Mladen Rogina

Lucia Romani

Milvia Rossini

James Rossmanith

Miroslav Rozložník

Siegfried M. Rump

Andreas Rössler

Giancarlo Sangalli
Tomas Sauer

J.M. Sanz-Serna

Driss Sbibih

Othmar Scherzer

Rene Schilling

Dominik Schoetzau

Meiyue Shao

Simon Shaw

Qin Sheng

Eric Sonnendrücker

Tanya Sorokina

Hendrik Speleers

Benjamin Stamm

Zdenek Strakos

Martin Stynes

Caren Tischendorf

Angel Tocino

Miroslav Tůma

Karsten Urban

Raf Vandebril

Olivier Verdier

Rossana Vermiglio

Stefan Volkwein

Heinrich Voss

Li-Lian Wang

Yoshitaka Watanabe

Rüdiger Weiner

Peter Wild

Joerg Willems

Bin Wu

Bernardo de la Calle Ysern

Jens-Peter M. Zemke

Shun Zhang

Ludmil Zikatanov

Thank you all for this year and see you later for BIT!

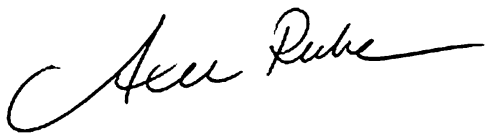

Axel Ruhe 